Pesq. Vet. Bras. 36(9):874-880, setembro 2016

DOI:10.1590/S0100-736X2016000900014

\title{
Quantificação de fenóis, flavonoides totais e atividades farmacológicas de geoprópolis de Plebeia aff. flavocincta do Rio Grande do Norte ${ }^{1}$
}

\author{
Jardel B. Silva2*, Kizzy M.F.M. Costa², Wesley A.C. Coelho ${ }^{3}$, Kaliane A.R. Paiva ${ }^{2}$, \\ Geysa A.V. Costa ${ }^{2}$, Antonio Salatino ${ }^{4}$, Carlos I.A. Freitas ${ }^{2}$ e Jael S. Batista ${ }^{2}$
}

\begin{abstract}
Silva J.B., Costa K.M.F.M., Coelho W.A.C., Paiva K.A.R., Costa G.A.V., Salatino A., Freitas C.I.A. \& Batista J.S. 2016. [Quantification of phenols, total flavonoids and pharmacological activities of geopropolis Plebeia aff. flavocincta of Rio Grande do Norte, Brazil.] Quantificação de fenóis, flavonoides totais e atividades farmacológicas de geoprópolis de Plebeia aff. flavocincta do Rio Grande do Norte. Pesquisa Veterinária Brasileira 36(9):874-880. Departamento de Ciências Animais, Universidade Federal Rural do SemiÁrido, Av. Francisco Mota, Presidente Costa e Silva, Mossoró, RN 59625-900, Brazil. E-mail: jardelbezerra@bol.com.br

This study aimed to evaluate the chemical composition and therapeutic potential of the hydroalcoholic extract of geopropolis produced by bees Plebeia aff. flavocincta in Rio Grande do Norte semi-arid. Eight samples (A-H) were submitted to the quantification of total phenols and flavonoid content and evaluation of the antioxidant activity by DPPH method (2,2-diphenyl-1-picryl-hydrazyl). Additionally, antibacterial activities were investigated by agar diffusion method, and healing process by inducing experimental surgical wounds in Rattus norvegicus Berkenhout, 1769, Wistar line, and treated with the geopropolis-based cream. Two samples (F and G) have stood out by presenting high values of total phenols and flavonoids. The antioxidant capacity of over $90 \%$ was observed in five samples. The extracts were considered effective in relation to antibacterial activity since of the eight samples, five promoted the formation of inhibition zones $\geq 9 \mathrm{~mm}$ above all strains tested. Two samples (D and G) have stood out over the other for presenting excellent results since it promoted the formation of inhibition zones for Staphylococcus epidermidis and Escherichia coli exceeding statistically to the values of the antibiotic controls when the concentration was $100 \%$. Samples A and G have provided, respectively, that the formation of inhibition zones were not significantly different than the halos produced by antibiotics controls at all concentrations tested for Escherichia coli and Staphylococcus aureus. The analysis of the healing process from the clinical, macroscopic and histological points of view, allowed to conclude that the use of geopropolis-based cream had a positive influence on the healing of experimental skin wounds by promoting less intense inflammatory reaction and healing faster from wounds in the control group.
\end{abstract}

INDEX TERMS: Phenols, flavonoids, geopropolis, Plebeia aff. flavocincta, stingless bees, therapeutic potential, semiarid region, Brazil.

${ }^{1}$ Recebido em 19 de janeiro de 2016.

Aceito para publicação em 22 de maio de 2016

${ }^{2}$ Departamento de Ciências Animais, Universidade Federal Rural do Semi-Árido (Ufersa), Av. Francisco Mota, Presidente Costa e Silva, Mossoró, RN 59625-900, Brasil. *Autor para correspondência: jardelbezerra@bol.com.br

${ }^{3}$ Faculdade de Enfermagem Nova Esperança de Mossoró (Facene), Av. Presidente Dutra, Alto de São Manoel, Mossoró, RN 59628-000.

${ }^{4}$ Departamento de Botânica, Universidade de São Paulo (USP), Rua do Matão, Cidade Universitária, Butantã, São Paulo, SP 05508-090, Brasil.
RESUMO.- Esse estudo objetivou avaliar a composição química e potencial terapêutico do extrato hidroalcóolico de geoprópolis produzidas por abelhas Plebeia aff. flavocincta no semiárido do Rio Grande do Norte. Oito amostras (AH) foram submetidas a quantificação dos teores de fenóis e flavonoides totais e avaliação da atividade antioxidante através do método DPPH (2,2-difenil-1-picril-hidrazilo). Além disso, foram investigadas as atividades antibacteria- 
na, através do método de difusão em ágar, e a cicatrizante, através da indução de feridas cirúrgicas experimentais em Rattus norvegicus Berkenhout, 1769, linhagem Wistar, tratados com creme à base de geoprópolis. Duas amostras (F e G) se destacaram por apresentar altos valores de fenóis e flavonoides totais. A capacidade antioxidante acima de $90 \%$ foi observada em cinco amostras. Os extratos foram considerados eficazes no que se refere a atividade antibacteriana, pois das oito amostras, cinco promoveram a formação de halos de inibição $\geq 9 \mathrm{~mm}$ para todas as cepas testadas. Duas amostras (D e G) se destacaram em relação as demais por apresentar excelentes resultados, uma vez que, promoveram a formação de halos de inibição para as bactérias Staphylococcus epidermidis e Escherichia coli, superiores estatisticamente aos valores dos antibióticos controles, quando na concentração de $100 \%$. As amostras A e G promoveram, respectivamente, a formação de halos de inibição, que não diferiram significativamente dos halos produzidos pelos antibióticos controles em todas as concentrações testadas para E. coli e Staphylococcus aureus. A análise do processo de cicatrização sob os pontos de vista clínico, macroscópico e histológico permitiu concluir, que o uso do creme à base de geoprópolis apresentou influência positiva na cicatrização de feridas cutâneas experimentais, por promover reação inflamatória menos intensa e fechamento mais rápido das feridas em relação ao grupo controle.

TERMOS DE INDEXAÇÃO: Fenóis, flavonoides, atividades farmacológicas, geoprópolis, Plebeia aff. flavocincta, Brazil, abelhas sem ferrão, potencial terapêutico, semiárido, Brasil.

\section{INTRODUÇÃO}

As abelhas da subfamília Meliponinae (Hymenoptera, Apidae) são conhecidas como abelhas indígenas sem ferrão, ou ainda meliponídeos, assim chamadas por possuírem o ferrão atrofiado. São abelhas sociais nativas de ampla distribuição geográfica no Brasil, com mais de 250 espécies, distribuídas em 27 gêneros (Silveira et al. 2002), apresenta importância econômica, ambiental e social dentro de diversos nichos e regiões onde ocorrem as abelhas (Nogueira-Neto 1997, Slaa et al. 2006).

Inicialmente desenvolvida pelos índios, a meliponicultura, ao longo do tempo foi praticada de forma tradicional por pequenos e médios produtores, principalmente por aqueles que usavam mão de obra familiar nas atividades agropecuárias, em diversas regiões do Brasil (Alves et al. 2007). Especialmente nas regiões Norte e Nordeste, constitui fonte adicional de renda, em algumas ocasiões é parte importante da agricultura familiar, pois seus produtos (mel, pólen, própolis e geoprópolis) são bastante apreciados pela população local devido seus valores nutricionais e terapêuticos, sendo valorizados economicamente (Souza et al. 2004, Lobato \& Venturieri 2010). A literatura destaca ainda o papel ecológico das abelhas brasileiras sem ferrão, uma vez que, são responsáveis por 40 a $90 \%$ da polinização das árvores nativas (Kerr et al. 1996).

Por serem sem ferrão e de um modo geral não agressivas, os meliponídeos são bastante aceitos nas propriedades rurais no Brasil (Rodrigues 2006). Referindo-se especial- mente ao Nordeste, há diversos polos bem-sucedidos de meliponicultura. Os meliponicultores exploram em cortiços ou em caixas racionais espécies produtivas, adaptadas às condições de vegetação e clima locais, como: Tiúba $(M e-$ lipona compressipes (Fabricius, 1804)) no Maranhão e Piauí (Carvalho-Zilse \& Kerr 2004, Monte et al. 2013), Jandaíra (Melipona subnitida Ducke) no Ceará (Cruz et al. 2004), Jandaíra e Mosquito (Plebeia sp.), Uruçu (Melipona scutellaris) em Pernambuco e Paraíba (Carvalho-Zilse et al. 2009, Gois et al. 2010) e Cupira (Partamona sp.) no Rio Grande do Norte (Pereira et al. 2011).

Dentre os produtos oriundos dos meliponídeos se destaca a geoprópolis, que tem suscitado crescente interesse dos pesquisadores, que vem desenvolvendo recentes estudos voltados para o conhecimento de sua composição química e atividades farmacológicas (Fianco et al. 2013, Lima 2015, Sousa et al. 2015). A geoprópolis é uma mistura complexa formada por resinas, obtidas pelas abelhas da flora da região (pasto apícola), misturada a cera e secreções salivares das abelhas, além de terra e/ou barro, a qual é utilizada na construção da entrada e das divisórias do local de nidificação da espécie, vedação de frestas e no reparo de danos à colmeia (Funari \& Ferro 2006, Carvalho-Zilse \& Nunes-Silva 2012).

A realização de pesquisa a respeito da constituição química da geoprópolis se torna indispensável para relacionar os compostos bioativos presentes em sua formulação, com suas atividades farmacológicas testadas, ampliando o respaldo da qualidade desses produtos e oferecendo garantia aos consumidores que fazem uso em sua rotina (Eduardo 2014). Em trabalho de levantamento das abelhas indígenas sem ferrão criadas em meliponários no Rio Grande do Norte, Pereira et al. (2011) observaram que o gênero Plebeia apresentou uma frequência de 4,9\%, no entanto, são inexistentes pesquisas que envolvam estudos voltados para composição química e atividades farmacológicas de geoprópolis da abelha Plebeia aff. flavocincta. Assim, esse estudo objetivou avaliar a composição química e potencial terapêutico do extrato hidroalcóolico de geoprópolis produzidas por abelhas Plebeia aff. flavocincta no semiárido do Rio Grande do Norte.

\section{MATERIAL E MÉTODOS}

Obtenções das amostras e preparação dos extratos. No período de julho a setembro de 2014, foram coletadas oito amostras de geoprópolis (A, B, C, D, E, F, G e H) produzidas por abelhas da espécie $P$. aff. flavocincta, junto à meliponicultores na zona rural de Serra do Mel e Mossoró, Rio Grande do Norte, Brasil. 50g das geoprópolis foram submetidas à secagem em estufa de circulação de ar a $40^{\circ} \mathrm{C}$ por $48 \mathrm{~h}$, trituradas em multiprocessador Britânia por cinco minutos, obtendo-se um pó fino e homogêneo. Esse material foi pesado e depositado em recipiente de vidro âmbar com adição de solução hidroalcóolica a 70\% na proporção de 1:3 do pó. A mistura foi mantida em temperatura ambiente durante 20 dias sob agitação frequente. Na etapa seguinte realizou-se processo de filtragem do sobrenadante em papel filtro, para retenção de partículas insolúveis.

Quantificação de fenóis totais. 0 teor de fenóis totais foi determinado empregando-se o método Waterman \& Mole (1994), em que alíquotas de $0,02 \mathrm{ml}$ em triplicata de cada extrato hidroalcóolicos de geoprópolis foram transferidas para um tubo de 
ensaio, adicionando-se 0,08ml de Metanol ( $\mathrm{MeOH})$ e em seguida, transferidos para balões volumétricos de $10 \mathrm{ml}$, contendo $3,9 \mathrm{ml}$ de água destilada. Adicionaram-se $0,75 \mathrm{ml}$ de carbonato de sódio $\left(\mathrm{Na}_{2} \mathrm{CO}_{3}\right)$ e $0,25 \mathrm{ml}$ do reagente Folin-Ciocalteau. Após $60 \mathrm{minu}-$ tos, realizou-se a leitura em espectrofotômetro (Coleman 33D) a 760nm, utilizando como padrão o ácido tânico, nas concentrações de $100,200,400,600,800,1000$ e $1200 \mathrm{mg} / \mathrm{ml}$ para construir uma curva de calibração. A partir da equação da reta obtida na curva do gráfico do padrão, realizou-se o cálculo do teor de fenóis totais, sendo os resultados expressos em porcentagem de ácido tânico por grama de matéria seca.

Quantificação de flavonoides totais. A determinação dos teores de flavonoides totais foi realizada segundo o método descrito por Woisky \& Salatino (1998), em que alíquotas de 0,5ml, em triplicata de cada amostra dos extratos hidroalcóolico de geoprópolis, foram adicionadas a um volume igual de solução metanólica de Cloreto de alumínio $5 \%\left(\mathrm{AlCl}_{3}\right)$. Após repouso por $15 \mathrm{minu}$ tos, realizou-se a leitura em espectrofotômetro (Coleman 33D) a $420 \mathrm{~nm}$. 0 conteúdo de flavonoides totais foi determinado usando uma curva padrão de quercetina nas concentrações de $0,25,50$, $75,100,125,150,175,200,250$ e $300 \mathrm{mg} / \mathrm{ml}$. A partir da equação da reta obtida na curva do gráfico do padrão, realizou-se o cálculo do teor de flavonoides totais, sendo os resultados expressos em mg de quercetina por $100 \mathrm{~g}$ de geoprópolis.

Atividade antioxidante in vitro. Para avaliação da atividade antioxidante dos extratos de geoprópolis, foi utilizado o método fotocolorimétrico in vitro do radical livre DPPH (2,2-difenil-1-picrilhidrazil) descrito por Mensor et al. (2001). Para a análise das amostras, adicionou-se $20 \mu \mathrm{g} / \mathrm{ml}$ da solução metanólica dos extratos diluídos a $220 \mu \mathrm{g} / \mathrm{ml}$ de uma solução metanólica de radical livre DPPH. Após 20 minutos de reação, realizou-se a leitura em 518nm, utilizando-se espectrofotômetro Ultravioleta UV-vis Shimadzu UV 1601. Todas as leituras foram realizadas em triplicata e com a média dos dados obtidos, foi calculado o percentual da atividade antioxidante dos extratos de geoprópolis, pela seguinte fórmula: $\mathrm{AA}=100-[(\mathrm{Aa}-\mathrm{Ab}) \times 100] / \mathrm{Ac}$. Onde $\% \mathrm{AA}=$ porcentagem de atividade antioxidante; $A a=$ absorbância da amostra; $A b=a b-$ sorbância do branco; $\mathrm{Ac}=$ absorbância do controle.

Atividade antibacteriana in vitro. Para avaliação da atividade antibacteriana dos extratos de geoprópolis foi utilizado o método de difusão em ágar Mueller Hinton (AMH), pela técnica de perfuração de poços (Ostrosky et al. 2008). A partir do extrato bruto, as amostras foram diluídas em etanol $96^{\circ} \mathrm{GL}$ nas concentrações de $100 \%, 75 \%, 50 \%, 25 \%$ e $12 \%$. As cepas utilizadas foram provenientes de coleções padronizadas pela American Type Cell Culture (ATCC), devidamente caracterizadas morfológica, fisiológica e bioquimicamente. As cepas testadas foram de Staphylococcus aureus (ATCC 6538), Staphylococcus epidermidis (ATCC 12228), Escherichia coli (ATCC 25922) e Pseudomonas aeruginosa (ATCC 35032). Como controle positivo utilizou-se ciprofloxacina $(5 \mu \mathrm{g})$ para bactérias gram-positivas ( $S$. aureus e $S$. epidermidis) e amoxicilina $(10 \mu \mathrm{g})$ para bactérias gram-negativas $(E$. coli e $P$. aeruginosa) e como controle negativo $50 \mu \mathrm{l}$ de álcool $70^{\circ} \mathrm{GL}$. Os resultados foram obtidos através da mensuração do diâmetro dos halos de inibição ao redor dos poços, com o auxílio de paquímetro e expressos em milímetros, de acordo a metodologia descrita por Lima et al. (2006). A presença de zonas de inibição de crescimento de $\geq 9,0 \mathrm{~mm}$ ao redor dos poços foi considerada como indicativo de atividade antibacteriana.

Atividade cicatrizante in vivo. Os efeitos do tratamento tópico do creme à base de geoprópolis foi avaliado utilizando-se 40 ratos (Rattus norvegicus Berkenhout, 1769), linhagem Wistar, machos, com 60 dias de idade, com peso corporal de 250g. Esses animais foram divididos em grupo experimental (GE), composto por 20 ratos com feridas cutâneas tratadas com aplicação tópica do creme-base com $20 \%$ de geoprópolis e grupo controle (GC), com o mesmo número de animais, tratados com aplicação tópica do creme-base sem geoprópolis. Após antissepsia e anestesia com cloridrato de xilazina $(8,0 \mathrm{mg} / \mathrm{kg}$, Xilazin, Syntec, Brasil) e cloridrato de cetamina $(140 \mathrm{mg} / \mathrm{kg}$, Cetamin, Syntec, Brasil) por via intramuscular, realizou-se uma lesão com $1 \times 1 \mathrm{~cm}$ de diâmetro centralizada na região dorsal. 0 creme foi aplicado imediatamente após cirurgia e posteriormente, uma vez ao dia com auxílio de espátulas de madeiras estéreis e individuais. As lesões foram avaliadas sob o aspecto clínico e morfométrico nos 3ํㅜ $7^{\circ}$ ํ, $14^{\circ}$ e e $21^{\circ}$ o dias após cirurgia, segundo recomendações de Prata et al. (1988). Para verificar microscopicamente a evolução da cicatrização, no mesmo período, foram realizadas biópsias das feridas cirúrgicas. Fragmentos obtidos foram fixados em formalina a 10\% e submetidos à inclusão em parafina, para obtenção de cortes de $5 \mu \mathrm{m}$ de espessura e corados pelo método de hematoxilina-eosina.

0 presente estudo foi realizado sob aprovação da Comissão de Ética em Experimentação Animal da Universidade Estadual do Rio Grande do Norte (CEEA), sob Parecer no. 1/15, tendo sido respeitados todos os preceitos éticos de proteção aos animais, conforme preconizado pelo Colégio Brasileiro de Experimentação Animal (COBEA) e pela Lei Auroca, no. 11.794.

Utilizou-se um computador com programa específico de análise de imagem (Image Pro Plus, versão 6.0 para Windows), no qual foi quantificada a densidade dos elementos histológicos, que indicam a evolução do processo cicatricial: células polimorfonucleares, células mononucleares, vasos sanguíneos, fibroblastos e fibras colágenas.

Análise estatística dos dados. Os dados foram expressos em valores de média e desvio padrão através do programa estatístico GrapPad Prism versão 5.0 e IBM SPSS Statistics for Windows (Armonk, NY: IBM Corp.) versão 22.0. Após análise dos pressupostos paramétricos, diferenças estatísticas das variáveis da densidade dos parâmetros histológicas entre grupos experimentais dentro de cada dia estudado, foram obtidas através de teste $t$ independente. Já diferenças entre os dias para cada grupo, utilizou-se análise de variância seguida por Tukey.

Em relação aos halos de inibição de crescimento bacteriano, diferença entre geoprópolis, dentro e entre concentrações (massa seca), foram obtidas por análise de variância. A influência das concentrações (massa seca) da geoprópolis frente atividade de fenóis totais, flavonoides e atividade antioxidante foram feitas através da correlação de Spearman. Por fim, elaborou-se um modelo de regressão linear múltipla, tendo como variável dependente a atividade antioxidante. Quando rompido distribuição gaussiana, utilizou-se transformação logarítmica. Variáveis percentuais sofreram transformação arcoseno. Valores de $p<0,05$ foram considerados significativos.

\section{RESULTADOS E DISCUSSÃO}

\section{Teor de fenóis e flavonoides totais}

As amostras F e G se destacaram com relação às demais, por apresentar, respectivamente, altos valores de fenóis totais $(5,67 \pm 0,39 \%$ e $9,40 \pm 0,28 \%)$ e maiores concentrações de flavonoides totais $(10,05 \mathrm{mg}$ e $22,00 \mathrm{mg} / 100 \mathrm{~g}$ de geoprópolis) (Quadro 1).

Vale salientar que esse é um estudo pioneiro, não sendo encontrados na literatura dados relativos à quantificação de fenóis e flavonoides totais da geoprópolis produzida pela abelha Plebeia aff. flavocincta. Em relação a outros meliponídeos, cujas informações podem ser comparadas às observações do presente estudo, merece destaque a pesquisa realizada por Franchin et al. (2012), que também ob- 
Quadro 1. Concentrações (massa seca) $\mathrm{mg} / \mathrm{ml}$, teor de fenóis totais $(\%)(\mathrm{m} / \mathrm{m})$, teor de flavonoides totais $(\mathrm{mg} / \mathbf{1 0 0 g})$ e atividade antioxidante $(\%)$ e seus respectivos desvios padrão dos oito extratos hidroalcoólicos da geoprópolis de Plebeia aff. flavocincta

\begin{tabular}{ccccc}
\hline Amostras & \multicolumn{4}{c}{ Análises } \\
\cline { 2 - 4 } & $\begin{array}{c}\text { Concentrações } \\
\text { Massa seca (mg/ml) }\end{array}$ & \multicolumn{2}{c}{ Teor } & $\begin{array}{c}\text { Atividade } \\
\text { antioxidante (\%) }\end{array}$ \\
\cline { 3 - 4 } & Fenóis totais (\%) & $\begin{array}{c}\text { Flavonoides totais } \\
(\mathrm{mg} / 100 \mathrm{~g})\end{array}$ & \\
\hline $\mathrm{A}$ & $33,43 \pm 0,53^{\mathrm{B}}$ & $2,67 \pm 0,21^{\mathrm{E}}$ & $4,1 \pm 0,07^{\mathrm{E}}$ & $94,31 \pm 1,12^{\mathrm{A}}$ \\
$\mathrm{B}$ & $18,95 \pm 0,36^{\mathrm{F}}$ & $0,99 \pm 0,09^{\mathrm{F}}$ & $1,48 \pm 0,07^{\mathrm{F}}$ & $89,72 \pm 2,38^{\mathrm{B}}$ \\
$\mathrm{C}$ & $21,6 \pm 0,24^{\mathrm{E}}$ & - & $0,5 \pm 0,03^{\mathrm{H}}$ & $24,45 \pm 1,50^{\mathrm{D}}$ \\
D & $32,53 \pm 0,25^{\mathrm{C}}$ & $3,13 \pm 0,24^{\mathrm{D}}$ & $6,18 \pm 0,30^{\mathrm{C}}$ & $94,97 \pm 0,39^{\mathrm{A}}$ \\
E & $26,45 \pm 0,30^{\mathrm{D}}$ & $4,01 \pm 0,55^{\mathrm{C}}$ & $4,6 \pm 0,00^{\mathrm{D}}$ & $93,79 \pm 0,34^{\mathrm{A}}$ \\
F & $34,08 \pm 0,66^{\mathrm{B}}$ & $5,67 \pm 0,39^{\mathrm{B}}$ & $10,05 \pm 0,04^{\mathrm{B}}$ & $95,65 \pm 0,05^{\mathrm{A}}$ \\
G & $37,60 \pm 0,44^{\mathrm{A}}$ & $9,4 \pm 0,28^{\mathrm{A}}$ & $22,00 \pm 0,37^{\mathrm{A}}$ & $95,46 \pm 0,10^{\mathrm{A}}$ \\
H & $33,68 \pm 0,68^{\mathrm{B}}$ & - & $0,8524 \pm 0,09^{\mathrm{G}}$ & $49,93 \pm 0,07^{\mathrm{C}}$
\end{tabular}

$\overline{\mathrm{A}, \mathrm{B}}$ Letras maiúsculas diferentes na coluna significa diferença estatística $(\mathrm{p}<0,05)$.

Quadro 2. Correlação entre concentrações (massa seca) mg/ml, atividade antioxidante (\%), fenóis totais (\%) (m/m) e flavonoides totais $(\mathrm{mg} / \mathbf{1 0 0 g}) \mathrm{dos}$ oito extratos hidroalcoólicos da Geoprópolis de Plebeia aff. flavocincta

\begin{tabular}{lccc}
\hline & Concentração & Atividade Antioxidante & Fenóis totais \\
\hline Atividade antioxidante & $0,828(<0,0001)^{* *}$ & - & \\
Fenóis totais & $0,841(<0,0001)^{* *}$ & $0,870(<0,0001)^{* *}$ & - \\
Flavonoides totais & $0,833(<0,0001)^{* *}$ & $0,912(<0,0001)^{* *}$ & $0,957(<0,0001)^{* *}$
\end{tabular}

** Significância estatística $(\mathrm{p}<0,001)$.

servaram altos teores de fenóis totais na geoprópolis produzido pela abelha Melipona scutellaris (uruçu).

\section{Atividade antioxidante}

Os resultados indicam que cinco amostras (A, D, E, F, G) apresentaram excelente atividade antioxidante, acima de 90\% (Quadro 1). Também se constatou correlação positiva entre atividade antioxidante e as variáveis fenóis totais $(\mathrm{rs}=0,870)$ e flavonoides totais $(\mathrm{rs}=0,912)$, ou seja, à medida que aumentaram os teores nas amostras, houve o aumento percentual da atividade antioxidante (Quadro 2). Tal fato confirma dados da literatura que afirma que a atividade antioxidante está diretamente correlacionada aos teores de fenóis e flavonoides totais (Castro et al. 2007, Cabral et al. 2009, Da Silva et al. 2013). Os compostos fenólicos são capazes de interceptar a cadeia de oxidação de radicais livres através da doação de hidrogênio de suas hidroxilas fenólicas (Righi et al. 2011). A constatação da atividade antioxidante nos extratos da geoprópolis poderá contribuir para validar seu uso medicinal, uma vez que, a utilização como remédio caseiro dos produtos elaborados pelos meliponídeos faz parte dos hábitos de muitas comunidades rurais no semiárido do nordeste brasileiro (Souza et al. 2004).

\section{Atividade antibacteriana}

0 extrato de geoprópolis da abelha $P$. aff. flavocincta foi considerado eficaz sob o ponto de vista da atividade antibacteriana, pois, das oito amostras, cinco (A, D, F, G e H) promoveram a formação de halos de inibição $\geq 9 \mathrm{~mm}$ para todas as cepas testadas. Na medida em que aumentou a concentração do extrato, houve ampliação dos halos de inibição do crescimento das bactérias nas placas, demonstrando ação concentração dependente para as bactérias gram-positiva e gram-negativa. Para as amostras D e G foram obtidos excelentes resultados, uma vez que, na concentração de $100 \%$, promoveram, respectivamente, a formação de halos de inibição para a bactéria S. epidermidis e E. coli, superiores estatisticamente aos os valores dos antibióticos controles. As amostras A e G proveram, respectivamente, a formação de halos de inibição, que não diferiram significativamente dos halos produzidos pelos antibióticos controles em todas as concentrações testadas para E. coli e S. aureus (Quadro 3).

Os resultados obtidos no presente estudo estão de acordo com a observação de Bankova (2005), no qual afirma que os diferentes tipos de própolis possuem atividade antibacteriana, pois as abelhas têm esse material como mais importante mecanismo de defesa química, utilizando contra microrganismos patogênicos e no preparo de locais assépticos para postura da abelha rainha. Assim como já demonstrado pela própolis das abelhas do gênero Apis, alguns estudos vêm comprovando a capacidade da geoprópolis produzida pelos meliponídeos em inibir o crescimento de bactérias patogênicas que adquirem com bastante frequência resistência aos antibióticos sintéticos e apresentam alto grau de patogenicidade para seres humanos e animais (Cunha et al. 2013, Fianco et al. 2013, Campêlo et al. 2015, Lima 2015, Sousa et al. 2015).

\section{Atividade cicatrizante}

$\mathrm{Na}$ avaliação da atividade cicatrizante do creme à base de geoprópolis da abelha $P$. aff. flavocincta foi possível observar durante o exame macroscópico, que até o $4^{\circ}$ dia pós-operatório as feridas da maioria dos animais do GE, apre- 
Quadro 3. Média \pm desvio padrão dos valores dos halos de inibição, em milímetro (mm) de diferentes diluições (\%) de extratos hidroalcóolicos de geoprópolis da abelha

Plebeia aff. flavocincta sobre o crescimento de Staphylococcus aureus, Escherichia coli, Pseudomonas aeruginosa e Staphylococcus epidermidis, pelo método de difusão em ágar. (CP: Controle positivo - Antibiótico); (E: Extratos hidroalcóolicos)

\begin{tabular}{|c|c|c|c|c|c|c|}
\hline \multirow[t]{2}{*}{$\mathrm{E}$} & \multicolumn{5}{|c|}{ Concentração do extrato \% } & \multirow[t]{2}{*}{ C.P** } \\
\hline & 100 & 75 & 50 & 25 & 12,5 & \\
\hline \multicolumn{7}{|c|}{ Staphylococcus aureus } \\
\hline A & $9,33 \pm 0,57^{\text {aA }}$ & $8,33 \pm 0,58^{\mathrm{aB}}$ & $*$ & $*$ & $*$ & $8,67 \pm 0,58^{\mathrm{aC}}$ \\
\hline B & * & * & * & * & * & $11,00 \pm 1,00^{\mathrm{AB}}$ \\
\hline $\mathrm{C}$ & $*$ & $*$ & $*$ & $*$ & $*$ & $10,00 \pm 0,00^{\mathrm{BC}}$ \\
\hline D & $11,67 \pm 1$ & $0 \pm 1,0$ & $9,33 \pm 0$ & $00 \pm 0,0$ & $6,00 \pm 0,00^{\mathrm{cAB}}$ & $10,00 \pm 0,00^{\mathrm{abBC}}$ \\
\hline $\mathrm{E}$ & * & $*$ & $*$ & $*$ & $*$ & $9,00 \pm 0,00^{\mathrm{C}}$ \\
\hline $\mathrm{F}$ & $9,67 \pm 0,58$ & $9,33 \pm 0,58^{\mathrm{abAB}}$ & $9,00 \pm 1,0$ & $3,33 \pm 5$ & $2,67 \pm 4,62^{\mathrm{bB}}$ & $10,50 \pm 0,50^{\mathrm{aAB}}$ \\
\hline G & $11,00 \pm 1$ & $11,33 \pm 2,08^{\mathrm{aA}}$ & $10,67 \pm 1$ & $10,00 \pm 2$ & $8,00 \pm 1,00^{\mathrm{aA}}$ & $11,50 \pm$ \\
\hline $\mathrm{H}$ & $10,00 \pm 1,00^{\mathrm{aA}}$ & $*$ & $*$ & $*$ & $*$ & $11,00 \pm 0,00^{\mathrm{aAB}}$ \\
\hline \multicolumn{7}{|c|}{ Escherichia coli } \\
\hline A & $13,33 \pm 1,53^{\mathrm{aA}}$ & $11,00 \pm 1,00^{\mathrm{abA}}$ & $10,00 \pm 0,00^{\mathrm{abA}}$ & $10,33 \pm 2,3$ & $10,67 \pm 2,89^{\mathrm{abA}}$ & $12,00 \pm 1,73^{a}$ \\
\hline B & $3,00 \pm 5,2$ & $2,67 \pm 4,62^{\mathrm{bB}}$ & $2,33 \pm 4,0$ & $2,67 \pm 4$ & $2,33 \pm 4,04^{\mathrm{bA}}$ & $14,00 \pm 0$ \\
\hline $\mathrm{C}$ & $11,67 \pm 0,5$ & $9,00 \pm 1,00^{\mathrm{bcA}}$ & $9,33 \pm 1,1$ & $8,67 \pm 1$ & $6,67 \pm 1,15^{\mathrm{cdA}}$ & $12,00 \pm 1,00^{\mathrm{aA}}$ \\
\hline D & $14,00 \pm 1,0$ & $11,67 \pm 1,53^{\mathrm{abA}}$ & $11,33 \pm 1$ & $10,00 \pm 0$ & $8,00 \pm 1,00^{\mathrm{bA}}$ & $11,00 \pm 0,00^{\mathrm{ab} A \mathrm{~B}}$ \\
\hline E & $13,67 \pm 2$ & $10,67 \pm 1,15^{\mathrm{abA}}$ & $10,67 \pm 1$ & $3,33 \pm 5$ & $3,00 \pm 5,20^{\mathrm{bA}}$ & $9,00 \pm 0,87^{\mathrm{abBC}}$ \\
\hline $\mathrm{F}$ & $12,67 \pm 2,08^{\mathrm{abA}}$ & $12,00 \pm 1,73^{\mathrm{abA}}$ & $11,67 \pm 1,5$ & $9,33 \pm 0,58^{\mathrm{abcA}}$ & $5,33 \pm 4,73^{\mathrm{bcA}}$ & $13,00 \pm 0,00^{\mathrm{aAB}}$ \\
\hline G & $15,33 \pm 3,21^{\mathrm{aA}}$ & $13,00 \pm 1,73^{\mathrm{abA}}$ & $12,00 \pm 1$ & $9,67 \pm 0$ & $9,00 \pm 1,00^{\mathrm{bA}}$ & $11,00 \pm 0,00^{\mathrm{bBC}}$ \\
\hline $\mathrm{H}$ & $10,67 \pm 0,58^{\mathrm{aA}}$ & $8,00 \pm 1,00^{\mathrm{abAB}}$ & $8,00 \pm 1,00^{\mathrm{abA}}$ & $7,00 \pm 1,0$ & $2,67 \pm 4,62^{\mathrm{bA}}$ & $11,00 \pm 0,00^{\mathrm{aBC}}$ \\
\hline \multicolumn{7}{|c|}{ Pseudomonas aeruginosa } \\
\hline A & $11,67 \pm 0,58^{\mathrm{bBCD}}$ & $10,00 \pm 1,00^{\mathrm{bBC}}$ & $9,67 \pm 0,58^{\text {मв }}$ & $9,00 \pm 0,00^{\text {मв }}$ & $2,67 \pm 4,62^{\mathrm{cB}}$ & 25,00 \\
\hline B & * & * & * & * & * & $20,00 \pm 1,00$ \\
\hline $\mathrm{C}$ & $8,67 \pm 1,53^{\mathrm{bD}}$ & $8,33 \pm 0,58^{\mathrm{bc}}$ & $*$ & $*$ & $*$ & $22,00 \pm 0,00^{\mathrm{a}}$ \\
\hline D & $15,67 \pm 1,15^{\text {bв }}$ & $13,33 \pm 1,15^{\mathrm{bB}}$ & $13,00 \pm 1,73^{\mathrm{bcAB}}$ & $10,00 \pm 1,73^{\mathrm{cdB}}$ & $8,67 \pm 0,58^{d A}$ & $21,00 \pm 0,00^{\mathrm{a}}$ \\
\hline $\mathrm{E}$ & $10,00 \pm 0,00^{\mathrm{aCD}}$ & $9,67 \pm 0,58^{\mathrm{aBC}}$ & $10,00 \pm 1,00^{\mathrm{aB}}$ & $*$ & $*$ & $9,00 \pm 0,00^{\mathrm{a}}$ \\
\hline $\mathrm{F}$ & $14,33 \pm 0,58^{\mathrm{abBC}}$ & $11,67 \pm 1,53^{\mathrm{bBC}}$ & $11,33 \pm 1,53^{\mathrm{bB}}$ & $10,00 \pm 1,00^{\mathrm{bB}}$ & $9,00 \pm 2,00^{\mathrm{bA}}$ & $18,00 \pm 0,00^{\mathrm{a}}$ \\
\hline G & $20,33 \pm 3,79^{\mathrm{aA}}$ & $18,33 \pm 3,79^{\mathrm{abA}}$ & $16,00 \pm 2,65^{\mathrm{abA}}$ & $14,00 \pm 2,00^{\mathrm{abcA}}$ & $12,33 \pm 2,52^{\mathrm{bcA}}$ & $21,00 \pm 0,00^{\mathrm{a}}$ \\
\hline $\mathrm{H}$ & $11,00 \pm 1,73^{\mathrm{bCD}}$ & $9,00 \pm 1,00^{\mathrm{bBC}}$ & $9,33 \pm 2,52^{\mathrm{bB}}$ & $*$ & $*$ & $20,00 \pm 0,00^{\mathrm{a}}$ \\
\hline \multicolumn{7}{|c|}{ Staphylococcus epidermidis } \\
\hline A & $10,67 \pm 1,15^{\mathrm{bBC}}$ & $9,33 \pm 0,58^{\mathrm{bcBC}}$ & $9,33 \pm 1,53^{\mathrm{bcBC}}$ & $8,33 \pm 0,58^{\mathrm{cB}}$ & * & 17,0 \\
\hline B & $*$ & $*$ & * & * & * & $20,00 \pm 0,00$ \\
\hline $\mathrm{C}$ & $9,00 \pm 1,00^{\mathrm{bC}}$ & $8,67 \pm 0,58^{\mathrm{bBC}}$ & $*$ & $*$ & $*$ & $25,00 \pm 0,00^{\mathrm{a}}$ \\
\hline D & $14,00 \pm 1,00^{\mathrm{aB}}$ & $11,33 \pm 0,58^{\mathrm{bB}}$ & $10,33 \pm 0,58^{\mathrm{bcBC}}$ & $8,67 \pm 0,58^{\mathrm{cdB}}$ & $8,00 \pm 1,00^{\mathrm{dA}}$ & $10,00 \pm 0,00^{\mathrm{bc}}$ \\
\hline $\mathrm{E}$ & $9,33 \pm 1,15^{\mathrm{bc}}$ & $8,33 \pm 0,58^{\mathrm{bcBC}}$ & $7,67 \pm 0,58^{\mathrm{cc}}$ & $*$ & $*$ & $21,00 \pm 0,00^{\mathrm{a}}$ \\
\hline $\mathrm{F}$ & $13,67 \pm 1,53^{\mathrm{aB}}$ & $11,67 \pm 1,15^{\mathrm{abAB}}$ & $12,00 \pm 2,00^{\mathrm{aB}}$ & $10,67 \pm 0,58^{\mathrm{abcAB}}$ & $3,33 \pm 5,77^{\mathrm{bcA}}$ & $16,00 \pm 0,00^{\mathrm{a}}$ \\
\hline G & $19,33 \pm 2,52^{\mathrm{aA}}$ & $17,33 \pm 3,51^{\mathrm{abA}}$ & $16,33 \pm 2,52^{\mathrm{abA}}$ & $11,33 \pm 2,31^{\mathrm{bcA}}$ & $9,00 \pm 1,73^{\mathrm{cA}}$ & $20,00 \pm 0,00^{\mathrm{a}}$ \\
\hline $\mathrm{H}$ & $10,33 \pm 0,58^{\mathrm{abBC}}$ & $5,00 \pm 4,36^{\mathrm{bC}}$ & * & * & * & $13,00 \pm 0,00^{\mathrm{a}}$ \\
\hline
\end{tabular}

a,b Letras minúsculas diferentes na linha $\mathrm{e}^{\mathrm{A}, \mathrm{B}}$ Maiúsculas diferentes na coluna significa diferença estatística $(\mathrm{p}<0,05)$. * Não houve crescimento de halos de inibição. ** controle positivo foi comparado somente na linha C.N (controle negativo - Álcool $70^{\circ} \mathrm{GL}$ ) não apresentou formação de halo de inibição em nenhum ensaio.

sentaram discreta hiperemia e edema dos bordos, enquanto que os animais do GC, apresentaram bordos edemaciados, elevados e com presença de exsudato serosanguinolento. Até o $8^{\circ}$ dia pós-operatório houve desprendimento espontâneo da crosta que recobria a superfície das feridas em todos os animais dos GE, sendo possível verificar que as feridas se apresentavam com marcada redução do tamanho original, secas, de coloração esbranquiçada no decorrer do processo cicatricial, enquanto que nos animais do GC as crostas se desprenderam no $11^{\circ}$ dia pós-operatório e as feridas se apresentavam visivelmente maiores com coloração rósea, brilhante e úmida. Quanto à área da ferida, verificou-se diminuição progressiva do tamanho ao longo do tempo, com diferença significativa, entre o GE e GC a partir do 9을. Nia. No $16^{\circ}$ dia as feridas dos animais do GE estavam completamente fechadas, enquanto que no GC, o fechamento das feridas ocorreu no $21^{\circ}$ dia (Fig.1).

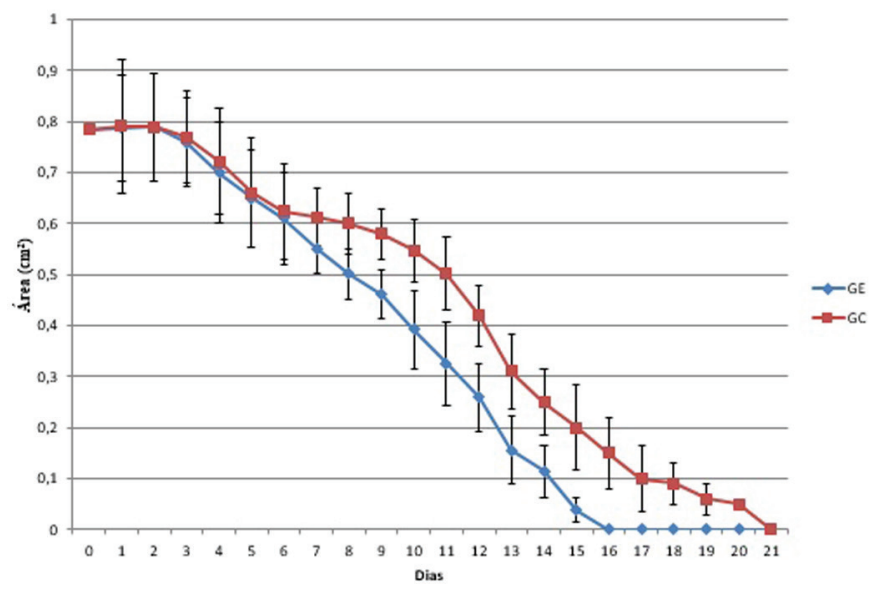

Fig.1. Gráfico do valor médio \pm desvio padrão das áreas das feridas em animais do Grupo Experimental (GE) e Grupo Controle (GC). 
Quadro 4. Valores de média \pm desvio padrão dos achados histológicos dos animais, pertencentes aos grupos controle (GC) e experimental (GE) nos dias 3, 7 e 14 pós-operatório

\begin{tabular}{lcccc}
\hline & Grupos & \multicolumn{3}{c}{ Período de observação (dias) } \\
\cline { 3 - 5 } & & $3^{\circ}$ & $7^{\circ}$ & $14^{\mathrm{o}}$ \\
\hline Polimorfo- & GE & $103456,68 \pm 64680,81^{\mathrm{aB}}$ & $453,00 \pm 172,08^{\mathrm{bB}}$ & $19,00 \pm 11,57^{\mathrm{bB}}$ \\
nucleares & GC & $219021,80 \pm 69107,13^{\mathrm{aA}}$ & $7210,00 \pm 3586,96^{\mathrm{bA}}$ & $350,00 \pm 2814,12^{\mathrm{bA}}$ \\
Vasos & GE & $13,80 \pm 2,77^{\mathrm{aA}}$ & $11,0 \pm 3,80^{\mathrm{aA}}$ & $4,20 \pm 2,48^{\mathrm{bA}}$ \\
Sanguíneos & GC & $7,60 \pm 5,31^{\mathrm{aA}}$ & $7,40 \pm 3,84 \mathrm{aA}$ & $3,20 \pm 1,09^{\mathrm{aA}}$ \\
Mononucleares & GE & $2,86 \pm 6,44^{\mathrm{cA}}$ & $9981,40 \pm 75596,99^{\mathrm{aA}}$ & $27509,60 \pm 18870^{\mathrm{bA}}$ \\
& GC & $89,60 \pm 15,40^{\mathrm{cA}}$ & $104052,40 \pm 34840,70^{\mathrm{aA}}$ & $3517,80 \pm 52521,90^{\mathrm{bA}}$ \\
Fibroblastos & GE & 0 & $249,80 \pm 57,44^{\mathrm{aA}}$ & $9469,60 \pm 28488,57^{\mathrm{bA}}$ \\
& GC & 0 & $39,46 \pm 28,48^{\mathrm{bB}}$ & $190,26 \pm 169,02^{\mathrm{aB}}$ \\
Colágeno & GE & 0 & $5312,20 \pm 2147,62^{\mathrm{bA}}$ & $29033,00 \pm 15419,25^{\mathrm{aA}}$ \\
& GC & 0 & $630,20 \pm 195,10^{\mathrm{bB}}$ & $918,42 \pm 97,91^{\mathrm{aB}}$
\end{tabular}

$\overline{\mathrm{a}, \mathrm{b}}$ Letras minúsculas diferentes na linha e $\mathrm{e}^{\mathrm{A}, \mathrm{B}}$ Maiúsculas diferentes na coluna significa diferença estatística $(\mathrm{p}<0,05)(\mathrm{GE})$ = Grupo experimental; $(\mathrm{GC})=$ Grupo controle.

A avaliação histológica das feridas cirúrgicas (Quadro 4 ), pode-se perceber o efeito benéfico do uso tópico do creme de geoprópolis da abelha $P$. aff. flavocincta, no qual foi constatado pela redução significativa da densidade de células polimorfonucleares, aumento significativo da contagem dos fibroblastos e fibras colágenas nos animais do GE. Os resultados das análises histológicas realizadas no presente estudo foram semelhantes aos obtidos por Berretta et al. (2012), que observaram diminuição do recrutamento de células inflamatórias e aumento da produção de colágeno em feridas cutâneas experimentais em ratos tratados com gel de própolis verde, após o terceiro dia de tratamento. Estudo realizado por Abreu et al. (2012) demonstraram que a aplicação tópica do extrato de própolis a $30 \%$ foi capaz de promover a fibroplasia, a re-epitelização e aceleração da cicatrização, quando comparada a um grupo controle.

Evidencias de edema e hiperemia discreta e a baixa densidade de células polimorfonucleares observadas nas feridas do GE e diferença estatística em relação ao GC, sugere uma atividade anti-inflamatória à geoprópolis da abelha $P$. aff. flavocincta. A inflamação é uma etapa fundamental ao processo cicatricial, pois promove a exsudação de células leucocitárias que realizam fagocitose e destroem agentes lesivos, restos tissulares e tecido necrótico (Mandelbaum et al. 2003). Contudo, com a persistência do processo inflamatório, se inicia a fase dos efeitos colaterais capazes de promover efeitos maléficos ao organismo (Robbins et al. 2009). 0 uso de cremes ou extratos de própolis tem sido amplamente testado na dermatologia no tratamento de feridas abertas, queimaduras e ulceras externas, com resultados promissores, por apresentar propriedades anti-inflamatórias (Moura et al. 2011, Pessolato et al. 2011).

Os resultados obtidos no presente estudo demonstraram que a geoprópolis da abelha $P$. aff. flavocincta se mostrou promissor sob o ponto de vista da composição química e atividade biológica. Todavia, vale salientar que a atividade antioxidantes e os teores de fenóis e flavonoides totais, frequentemente avaliados em pesquisa com geoprópolis, está intimamente relacionada com a flora de cada região visitada pelas abelhas e, portanto, pode ser influenciado por fatores climáticos, tais como temperatura, intensidade de luz e pluviosidade (Dutra et al. 2008, Cardozo et al. 2015,
Lima 2015). No semiárido de Mossoró e Serra do Mel, Rio Grande do Norte, locais de coleta das amostras, há predominância de Caatinga, que por ser de natureza caducifólia concorre para a grande flutuação da disponibilidade de alimentos para abelhas. Assim, a sazonalidade pode dificultar a padronização de extratos naturais para uso medicinal. Deste modo, são necessários estudos sazonais sobre a composição química e atividades biológicas da geoprópolis produzida por meliponídeos da Caatinga.

\section{CONCLUSÕES}

Foram detectados em extratos hidroalcóolico de geoprópolis da abelha $P$. aff. flavocincta, produzidas no semiárido do Rio Grande do Norte, altos teores de fenóis e flavonoides totais e capacidade antioxidante. Nas condições experimentais utilizadas, evidenciou-se atividade antibacteriana em cinco das oito amostras, frente a todas as cepas testadas.

0 creme à base de geoprópolis apresentou influência positiva na cicatrização de feridas cutâneas experimentais.

\section{REFERÊNCIAS}

Abreu A.M., Oliveira D.W.D., Marinho S.A., Lima N.L., Miranda J.L. \& Verli F.D. 2012. Effect of topical application of different substances on fibroplasia in cutaneous surgical wounds. ISRN Dermatol. 2012:1-5.

Alves R.M.O., Carvalho C.A.L. \& Souza B.A. 2007. Notas sobre a Bionomia de Melipona mandacaia (Apidae: Meliponina). Magistra 19(3):204-212.

Bankova V. 2005. Recent trends and important developments in propolis research. Evid. Based. Complement. Alternat. Med. 2(1):29-32.

Berretta A.A., Nascimento A.P., Bueno P.C.P., Vaz M.M.O.L.L. \& Marchetti J.M. 2012. Propolis standardized extract (EPP-AF®), an innovative chemically and biologically reproducible pharmaceutical compound for treating wounds. Int. J. Biol. Sci. 8(4):512-521.

Cabral I.S.R., Oldoni T.L.C., Prado A., Bezerra R.M.N., Alencar S.M., Ikegaki M. \& Rosalen P.L. 2009. Composição fenólica, atividade antibacteriana e antioxidante da própolis vermelha brasileira. Quím. Nova 32(6):1523-1527.

Campêlo M.C.S., Freire D.A.C., Abrantes M.R., Sousa E.S. \& Silva J.B.A. 2015. Potencial antimicrobiano de própolis e cera de diferentes espécies de abelhas sem ferrão. Acta Vet. Bras. 9(4):397-400.

Cardozo D.V., Mokochinski J.B., Schineider C.M., Sawaya A.C.H.F., Caetano I.K., Felsner M.L. \& Torres Y.R. 2015. Variabilidade química de geoprópolis produzida pelas abelhas sem ferrão Jataí, Mandaçaia e Mandurí. Revta Virtual Quim. 7(6):2457-2474.

Carvalho-Zilse G.A., Costa-Pinto M.F.F., Nunes-Silva C.G. \& Kerr W.E. 2009. 
Does beekeeping reduce genetic variability in Melipona scutellaris (Apidae, Meliponini)? Genet. Mol. Res. 8(2):758-765.

Carvalho-Zilse G.A. \& Kerr W.E. 2004. Substituição natural de rainhas fisogástricas e distância de vôo dos machos em Tiuba (Melipona compressipes fasciculata Smith, 1854) e Uruçu (Melipona scutellaris Latreille, 1811) (Apidae, Meliponini). Acta Amaz. 34(4):649-652.

Carvalho-Zilse G.A. \& Nunes-Silva C.G. 2012. Threats to the Stingless Bees in the brazilian Amazon: How to deal with scarce biological data and an increasing rate of destruction, p.147-168. In: Florio R.M. (Ed.), Bees: Biology, Threats and Colonies. Nova Science Publishers, New York.

Castro M.L., Cury J.A., Rosalen P.L., Alencar S.M., Ikegaki M., Duarte S. \& Koo H. 2007. Própolis do sudeste e nordeste do Brasil: influência da sazonalidade na atividade antibacteriana e composição fenólica. Quím. Nova 30(7):1512-1516.

Cruz D.O., Freitas B.M., Silva L.A., Silva E.M.S. \& Bomfim I.G.A. 2004. Adaptação e comportamento de pastejo da abelha jandaíra (Melipona subnitida Ducke) em ambiente protegido. Acta Sci. Anim. Sci. 26(3):293-298.

Cunha M.G., Franchin M., Galvão L.C.C., Ruiz A.L.T.G., Carvalho J.E., Ikegaki M., Alencar S.M., Koo H. \& Rosalen P.L. 2013. Antimicrobial and antiproliferative activities of stingless bee Melipona scutellaris geopropolis. BMC Complement. Altern. Med. 13(23):1-9.

Da Silva E.C.C., Muniz M.P., Nunomura R.C.S., Nunomura S.M. \& Zilse G.A.C. 2013. Constituintes fenólicos e atividade antioxidante da geoprópolis de duas espécies de abelhas sem ferrão amazônicas. Quim. Nova 36(5):628-633.

Dutra R.P., Nogueira A.M.C., Marques R.R.O., Costa M.C.P. \& Ribeiro M.N.S. 2008. Avaliação farmacognóstica de geoprópolis de Melipona fasciculata Smith da Baixada maranhense, Brasil. Rev. Bras. Farmacogn. 18(4):557562.

Eduardo L.F.P. 2014. Isolamento e identificação de compostos bioativos da geoprópolis (Melipona scutellaris) bioguiado pelo efeito antimicrobiano. Dissertação de Mestrado. Disponível em <http://www.bibliotecadigital. unicamp.br/document/?code $=000940862 \&$ opt $=2>$ Acesso em 10 maio 2016.

Fianco A.L.B., Falcão M.A., Cassel E. \& Milão D. 2013. Determinação da atividade antimicrobiana e teor de polifenóis totais de extratos etanólicos de própolis das abelhas sem ferrão Tetragonisca angustula (Jataí) e Scaptotrigona bipunctata (Tubuna). Revta Liberato 14(21):1-112.

Franchin M., Cunha M.G., Denny C., Napimoga M.H., Cunha T.M., Koo H., Alencar S.M., Ikegaki M. \& Rosalen P.L. 2012. Geopropolis from Melipona scutellaris decreases the mechanical inflammatory hypernociception by inhibiting the production of IL-1 beta and TNF-alpha. J. Ethnopharmacol. 143(2):709-715.

Funari C.S. \& Ferro V.O. 2006. Análise de própolis. Ciênc. Tecnol. Aliment. 26(1):171-178.

Gois G.C., Carneiro G.G., Rodrigues A.E., Silva E.O. \& Campos F.S. 2010. Qualidade microbiológica do mel de abelhas Melipona scutellaris. Pubvet 4(9):1-6.

Kerr W.K., Carvalho G.A. \& Nascimento V.A. 1996. Abelha Uruçu: biologia, manejo e conservação. Acangaú, Belo Horizonte, p.1-157.

Lima M.V.D. 2015. Geoprópolis produzida por diferentes espécies de abelhas: atividades antimicrobiana e antioxidante e determinação do teor de compostos fenólicos. Dissertação de Mestrado. Disponível em $<$ http://www.ppgcf.propesp.ufpa.br/ARQUIVOS/dissertacoes/marcuslima.pdf> Acesso em 10 mai. 2016.

Lima M.R.F., Ximenes C.P.A., Luna J.S. \& Sant'Ana A.E.G. 2006. The antibiotic activity of some Brazilian medicinal plants. Revta Bras. Farmacogn. 16(3):300-306.
Lobato T. \& Venturieri G.C. 2010. Aspectos econômicos da criação de abelhas indígenas sem ferrão (Apidae: Meliponini) no nordeste paraense. Embrapa Amazônia Oriental, Belém, p.1-36.

Monte A.M., Azevedo M.L.X., Cardoso Filho F.C., Rodrigues A.M.D., Moura S.G. \& Muratori M.C.S. 2013. Qualidade de méis de abelhas nativas sem ferrão do estado do Piauí, Brasil. Revta Bras. Med. Vet. 35(1):48-54.

Mandelbaum S.H., Santis E.P. \& Mandelbaum M.H.S. 2003. Cicatrization: current and auxiliary resources. Part 1. An. Bras. Dermatol. 78(4):393410.

Mensor L.L., Menezes F.S., Leitão G.G., Reis A.S., Santos T.C., Coube C.S. \& Leitão S.G. 2001. Screnning of Brazilian plant extracts for antioxidant activity by the use of DPPH free radical method. Phytother. Res. 15(2):127-130.

Moura S.A.L., Ferreira M.A.N.D., Andrade S.P., Reis M.L.C., Noviello M.L. \& Cara D.C. 2011. Brazilian green propolis inhibits inflammatory angiogenesis in a murine sponge model. Evid. Based Complement. Alternat. Med. 2011:1-7.

Nogueira-Neto P. 1997. Vida e Criação de Abelhas Indígenas sem Ferrão. Tecnapis, São Paulo, p.1-447.

Ostrosky E.A., Mizumoto M.K., Lima M.E.L., Kaneko T.M., Nishikawa S.O. \& Freitas B.R. 2008. Métodos para avaliação da atividade antimicrobiana e determinação da concentração mínima inibitória (CMI) de plantas medicinais. Revta Bras. Farmacogn. 18(2):301-307.

Pereira D.S., Menezes P.R., Belchior Filho V., Sousa A.H. \& Maracajá P.B. 2011. Abelhas indígenas criadas no Rio Grande do Norte. Acta Vet. Bras. 5(1):81-91.

Pessolato A.G.T., Martins D.S., Ambrósio C.E., Mançanares C.A.F. \& Carvalho A.F. 2011. Propolis and amnion reepithelialise second-degree burns in rats. Burns 37(70):1192-1201.

Prata M.B., Haddad C.M., Goldenberg S., Simöes M.J., Moura L.A.R. \& Trabulsi L.R. 1988. Uso tópico do açúcar em ferida cutânea: estudo experimental em rato. Acta Cir. Bras. 3(2):43-48.

Righi A.A., Alves T.R., Negri G., Marques L.M., Breyer H. \& Salatino A. 2011. Brazilian red propolis: unreported substances, antioxidant and antimicrobial activities. J. Sci. Food. Agric. 91(13):2363-2370.

Robbins S.L., Cotran R.S. \& Kumar V. 2009. Patologia estrutural e funcional. 6th ed. Guanabara Koogan, Brasil, Rio de Janeiro.

Rodrigues A.S. 2006. Até quando o etnoconhecimento sobre as abelhas sem ferrão (Hymenoptera, Apidae, Meliponinae) será transmitido entre gerações pelos índios Guarani M’byá da Aldeia Morro da Saudade, localizada na cidade de São Paulo, Estado de São Paulo, Brasil? Sitientibus Ser. Ciênc. Biol. 6(4):343-350.

Silveira F.A., Melo G.A.R. \& Almeida E.A.B. 2002. Abelhas Brasileiras: sistemática e identificação. Fundação Araucária, Belo Horizonte, p.1-253.

Slaa E.J., Sánchez-Chaves L.A., Malagodi-Braga K.S. \& Hofstede F.E. 2006. Stingless bees in applied pollination: practice and perspectives. Apidologie 37(2):293-315.

Sousa D.M.N., Olinda R.G., Martins C.G., Abrantes M.R., Coelho W.A.C., Silva J.B.A., Morais S.M. \& Batista J.S. 2015. Prospecção fitoquímica, toxicidade in vitro e avaliação das atividades anti-radicalar e antibacteriana da geoprópolis da abelha jandaíra. Acta Vet. Bras. 9(2):134-140.

Souza B.A., Carvalho C.A.L., Sodré G.S. \& Marchini L.C. 2004. Características físico-químicas de amostras de mel de Melipona asilvai (Hymenoptera: Apidae). Ciêncla Rural 34(5):1623-1624.

Waterman P.G. \& Mole S. 1994. Analysis of phenolic plant metabolites. Blackwell Scientific Publications 38(4):1064.

Woisky R.G. \& Salatino A. 1998. Analysis os propolis: some parameters ond prodecore for chemical fuality control. J. Apic. Res. 37(2):99-105. 